\title{
Joint Optimization of Electric Vehicle and Home Energy Scheduling Considering User Comfort Preference
}

\author{
Duong Tung Nguyen and Long Bao Le, Senior Member, IEEE
}

\begin{abstract}
In this paper, we investigate the joint optimization of electric vehicle (EV) and home energy scheduling. Our objective is to minimize the total electricity cost while considering user comfort preference. We take both household occupancy and $\mathrm{EV}$ travel patterns into account. The novel contributions of this paper lie in the exploitation of EVs as dynamic storage facility as well as detailed modeling of user comfort preference, thermal dynamics, EV travel, and customer occupancy patterns in a concrete optimization framework. Extensive numerical results are presented to illustrate the efficacy of the proposed design. Specifically, we show that the proposed design can achieve significant saving in electricity cost, allow more flexibility in setting the tradeoff between cost and user comfort, and enable to reduce energy demand during peak hours. We also demonstrate the benefits of applying the proposed framework to a residential community compared to optimization of individual household separately.
\end{abstract}

Index Terms-Electric vehicle, HVAC system, energy management system, aggregator, day-ahead electricity price, occupancy pattern, travel pattern, cost minimization, user comfort.

\section{NOMENCLATURE}

\section{$\Delta T \quad$ Duration of time slot (hours).}

$\delta_{k} \quad$ Maximum acceptable temperature deviation when house $k$ is occupied $\left({ }^{\circ} \mathrm{C}\right)$.

$\eta_{k} \quad$ Coefficient of performance (COP) of heater/AC in house $k$.

$\eta_{k, j}^{c} \quad$ Charging efficiency of $\mathrm{EV} j_{k}$.

$\eta_{k, j}^{\mathrm{d}} \quad$ Discharging efficiency of EV $j_{k}$.

$\Lambda_{k, j, l} \quad$ Travel time of EV $j_{k}$ during trip $l$.

$\Phi_{i}^{\mathrm{s}} \quad$ Solar irradiance in time slot $i\left(\mathrm{~kW} / \mathrm{m}^{2}\right)$.

$\sigma_{k} \quad$ Dummy variable, " -1 " for AC cooling, " 1 " for heating.

$A_{k} \quad$ The effective window area of house $k$.

Manuscript received November 22, 2012; revised May 07, 2013; accepted July 18, 2013. Date of publication September 12, 2013; date of current version December 24, 2013. Paper no. TSG-00810-2012.

The authors are with the Institut National de la Recherche Scientifique-Énergie, Matériaux et Télécommunications (INRS-EMT), Université du Québec, Montréal, QC H5A 1K6, Canada (e-mail: duong.nguyen@emt.inrs.ca; long. le@emt.inrs.ca).

Color versions of one or more of the figures in this paper are available online at http://ieeexplore.ieee.org.

Digital Object Identifier 10.1109/TSG.2013.2274521 $a_{k, i} \quad$ Occupancy state of house $k$ in time slot $i$, " 1 " for occupied, " 0 " otherwise.

$b_{k, j, i} \quad$ Availability state of $\mathrm{EV} j_{k}$ in time slot $i$, " 1 " for parking at home, "0" otherwise.

$C \quad$ Thermal capacitance $\left(\mathrm{kWh} /{ }^{\circ} \mathrm{C}\right)$.

$d_{k, j, l} \quad$ Travel distance of EV $j_{k}$ during trip $l$ (mile).

$e_{i} \quad$ Electricity price at time slot $i(\$ / \mathrm{kWh})$.

$E_{k, j}^{\text {cap }} \quad$ Battery capacity of EV $j_{k}(\mathrm{kWh})$.

$i$

$J_{k} \quad$ Number of EVs in house $k$.

$k \quad$ Household index, $k=1,2, \ldots, \mathrm{N}$.

$M \quad$ Number of households.

$m_{k, j} \quad$ Travel efficiency of EV $j_{k}(\mathrm{kWh} / \mathrm{mile})$.

$N \quad$ Number of time slots.

$P_{i}^{\text {grid }} \quad$ Imported power from the grid during time slot $i$ $(\mathrm{kW})$.

$P_{k}^{\text {hvac,max }}$ Power rating of heater/AC in house $k(\mathrm{~kW})$.

$P_{k, i}^{\text {hvac,out }}$ Output power of heater/AC in house $k$ at time slot $i(\mathrm{~kW})$.

$P_{k, i}^{\text {hvac }} \quad$ Power supplied to heater/AC in house $k$ at time slot $i(\mathrm{~kW})$.

$P_{k, j, i}^{\mathrm{ev}, c} \quad$ Charging power of EV $j_{k}$ at time slot $i(\mathrm{~kW})$.

$P_{k, j, i}^{\mathrm{ev}, d} \quad$ Discharging power of EV $j_{k}$ at time slot $i(\mathrm{~kW})$.

$P_{k, j}^{\mathrm{ev}, c, \max }$ Maximum charging power of EV $j_{k}(\mathrm{~kW})$.

$P_{k, j}^{\mathrm{ev}, d, \max }$ Maximum discharging power of EV $j_{k}(\mathrm{~kW})$.

$p_{k}^{\mathrm{s}} \quad$ The fraction of solar radiation entering the inner walls and floor.

$R \quad$ Thermal resistance between two heat exchange media $\left({ }^{\circ} \mathrm{C} / \mathrm{kW}\right)$.

$S O C_{k, j, i}$ State of charge of EV $j_{k}$ at time slot $i$.

$S O C_{k, j}^{\max }$ Maximum allowable state of charge of EV $j_{k}$.

$S O C_{k, j}^{\min }$ Minimum allowable state of charge of EV $j_{k}$.

$T_{i}^{\mathrm{a}} \quad$ Ambient temperature in time slot $i\left({ }^{\circ} \mathrm{C}\right)$. 
$T_{k, i}^{\mathrm{d}} \quad$ Desired indoor temperature of house $k$ in time slot $i\left({ }^{\circ} \mathrm{C}\right)$.

$T_{k, i}^{\mathrm{e}} \quad$ The temperature of the house envelope $\left({ }^{\circ} \mathrm{C}\right)$.

$T_{k, i}^{\text {in }} \quad$ Indoor temperature of house $k$ in time slot $i\left({ }^{\circ} \mathrm{C}\right)$.

$T_{k, i}^{\mathrm{m}} \quad$ The temperature of the thermal accumulating layer in the inner walls and floor $\left({ }^{\circ} \mathrm{C}\right)$.

$t_{k, j, l}^{(1)} \quad$ Time slot index when EV $j_{k}$ leaves home for trip $l$.

$t_{k, j l}^{(2)} \quad$ Time slot index when EV $j_{k}$ returns home for trip $l$.

$w_{k} \quad$ Weighting coefficient between electricity cost and discomfort cost for house $k\left(\$ /{ }^{\circ} \mathrm{C}\right)$.

\section{INTRODUCTION}

D EMAND SIDE management in the residential sector (i.e., residential buildings) is an important research topic since buildings contribute a significant fraction of overall electricity consumption. In fact, it accounted for $72 \%$ of total U.S. energy consumption in 2006 out of that residential buildings accounted for $51 \%$ according to the U.S. Environmental Protection Agency (EPA) [1]. This research topic has received lots of attention from the research community [2]-[8]. In [2], Shengnan et al. assessed the use of demand response as a load shaping tool to improve the distribution transformer utilization and avoid overloading for the transformer. Mohsenian-Rad et al. [3] proposed an optimization framework that aims to minimize electricity bills considering user comfort. However, the assumption on homogeneous appliances and using waiting time to represent user comfort in this paper would be too simple to represent different characteristics of home appliances and user requirements.

In a typical household, thermostatically controlled appliances (TCAs) including refrigerator, electric water heater, and the heating, ventilation, and air-conditioning (HVAC) system account for more than half of total residential energy consumption [1]. Research on optimal control for TCA loads has been a hot research topic in the last several years. References [4] and [5] proposed optimal control schemes to minimize the electricity cost for the HVAC system considering user climate comfort. Dynamic programming was employed in [6] to compare several optimal control algorithms applied to a thermostat. In [7], the authors introduced an appliance commitment algorithm that schedules electric water heater power consumption to minimize user payment.

Electric vehicle (EV) is another important grid element that has significant economic and environmental advantages compared to normal cars. The penetration of EVs is expected to increase drastically in the next few years, which can reach one million by 2015 in US [9]. Therefore, EV charging will have significant impacts on the power distribution network if it is not controlled appropriately [10]-[12]. EV travel pattern is an important factor to model potential impacts of EVs on the grid [13], [14] and to develop efficient EV charging strategies [15]. Given electricity prices and EV driving pattern, Rotering et al. proposed a dynamic programming based control scheme to optimize the charging for one EV [16]. In [17], Wu et al. considered load scheduling and dispatch problem for a fleet of EVs in both the day-ahead market and real-time energy market. In [18], an optimal charging strategy for EVs was proposed that considers voltage and power constraints.

The problems of scheduling of home energy usage and EV charging are often addressed separately in the literature. In this paper, we propose a unified optimization model that jointly optimizes the scheduling of EVs and TCAs. In particular, we utilize EVs as dynamic storage facility to supply energy for residential buildings during peak hours where energy can be transferred from EVs to charge other EVs and to provide energy for HVAC in a residential community. Our proposed model aims to minimize the total electricity cost considering user comfort, house occupancy and EV travel patterns, thermal dynamics, EV electricity demand, and other operation constraints. There are some recent works that discuss potential benefits of vehicle to building interactions [19], [20]. However, to the best of our knowledge, none of previous works have considered detailed design and joint optimization of EV and building energy management. The main contributions of this paper can be summarized as follows:

- We propose a comprehensive model to optimize the EV and HVAC scheduling in a residential area. The formulation aims to achieve flexible tradeoff between minimizing total electricity cost and maintaining user comfort preference. The model accounts for the characteristics of the HVAC system, thermal dynamics, user climate comfort preference, battery state model, user travel patterns, and household occupancy patterns. We also discuss potential extensions of the proposed framework to capture various modeling uncertainty factors.

- We show the impacts of different design and system parameters, which control the electricity cost and user comfort, on the system operation and performance as well as the economic benefits of applying our proposed control framework compared to a non-optimized control scheme for a single-house scenario.

- We illustrate the advantages of applying the proposed control model for the multiple-house scenario compared to the case where each household optimizes its energy consumption separately. Specifically, we demonstrate that optimization of EV and home energy scheduling for multiple houses in a residential community can achieve the significant saving in electricity cost and reduce the high power demand during peak hours.

The remaining of this paper is organized as follows. The system model is presented in Section II. Thermal dynamics model is described in Section III. Detailed problem formulation is described in Section IV. The case studies and numerical results are provided in Section V followed by conclusion in Section VI.

\section{System Model}

We consider the interaction among EVs and HVAC systems in the residential community. We assume that there is an aggregator that collects all required information from EVs and HVAC systems from all households to make control decisions. At the higher level, several aggregators can be connected to a central aggregator that coordinates the overall operations and participates in the wholesale day-ahead electricity market. The day-ahead market clearing price is assumed to be available to 


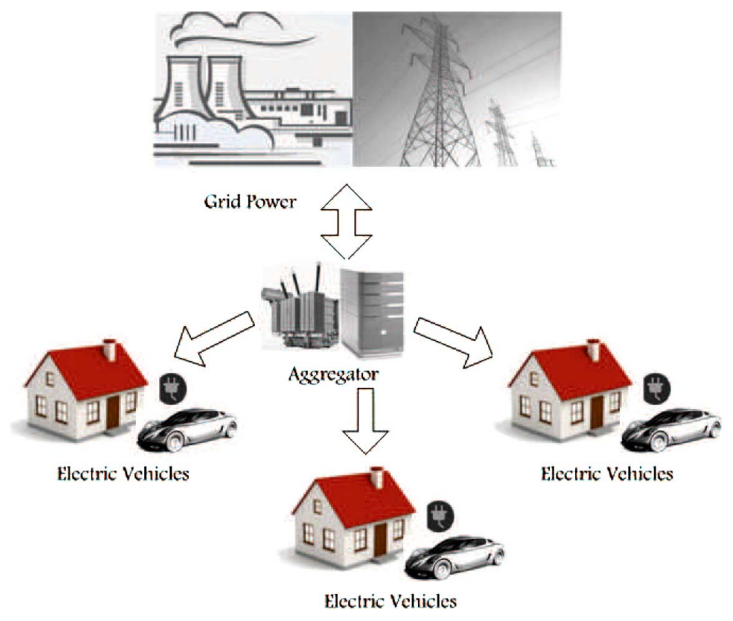

Fig. 1. System model.

any aggregators before the operating day. The system model under consideration is illustrated in Fig. 1.

We consider a time slotted model where there are $N$ time slots in the optimization period (e.g., 24 hours for one-day optimization period) and energy scheduling decisions are made for each time slot. ${ }^{1}$ Based on the pricing information and electricity demand, each aggregator decides how much energy it should import from the grid at each time slot and how to allocate and schedule the energy usage and to exchange energy among its components including HVAC and EVs. We assume that EVs can only be charged or discharged when they are parked at home (i.e., each household is equipped with the charging facility).

The slow thermal dynamic characteristics of buildings provides a great opportunity for demand side management since the building mass can be considered as a thermal storage facility. In particular, we can schedule the power consumption of HVAC systems flexibly while respecting users climate comfort because the indoor temperature changes quite slowly. By cooperating the energy scheduling of HVAC systems and EVs, it is expected that larger cost saving can be achieved compared to the case where we schedule these loads separately. Specifically, during high price hours, the energy discharging from an EV could be used to supply for other EVs and HVAC systems or can be sold back to the main grid. In the case we do not allow to sell EV discharging power back to the grid, the discharging power from EVs is assumed to only flow within the community network. In this paper, the term $\mathrm{V} 2 \mathrm{G}$ (vehicle-to-grid) refers to the case where selling back electricity to the main grid is allowed. ${ }^{2}$

\section{Building Thermal Dynamic Model}

To formulate the HVAC and EV scheduling problem, we need to model the dynamics of indoor air temperature and the characteristics of HVAC load in each household. In fact, modeling thermal dynamics of buildings is an important research topic that has been extensively studied in the literature. Among existing modeling methods for building thermal dynamics, the

\footnotetext{
${ }^{1}$ The thermal dynamics and energy scheduling model that we consider in this paper is in discrete-time, which is commonly assumed in the literature. Under this model, we assume that the thermal dynamics can reach its steady state in each time slot quickly so that the corresponding transient time can be neglected.

${ }^{2}$ In general, V2G can refer to the case where EVs are allowed to discharge energy regardless of whether the premises become a net supplier to the main grid.
}

grey-box approach appears to be one of the most popular ones. In this approach, we combine the physical knowledge about the building and experimental data to obtain a reasonable model for the building thermal dynamics [21]-[27]. Based on the energy balance and mass balance equations for the indoor air, a continuous time linear state space model, which is a set of first-order differential equations, can be constructed [26]-[28]. It is also called equivalent thermal parameter model (ETP) [7]. Then, experimental data is used to estimate building thermal parameters of the constructed model [23]-[27].

In the following, we present a thermal dynamic model for a residential housing. For household $k$, the indoor temperature can be expressed as a function of the housing thermal characteristics (thermal resistance, thermal capacitance, window area), the weather condition (ambient temperature, solar radiation, wind speed, humidity), internal gains (occupants, cooking, refrigerator, etc.), and the HVAC output power. Hence, we have

$T_{k, i+1}=f\left(T_{k, i}, P_{k, i}^{\mathrm{hvac}, \text { out }}\right.$, other inputs and parameters $)$.

For simplicity, we make the following assumptions.

- Each house is modeled as a large room exchanging thermal energy with the ambient environment. The indoor temperature is uniformly distributed within a house, which can be considered as the equivalent indoor temperature.

- If the household has more than one AC/heater (e.g., one $\mathrm{AC} /$ heater for each room) then the thermal output power from these $\mathrm{ACs} /$ heaters are gathered and considered as one aggregate $\mathrm{AC} /$ heater with the output power equal to the total output power of individual ACs/heaters [22], [23]. In practice, a central HVAC system can provide all cooling/ heating loads of buildings [28], [29].

- The impact of disturbances such as humidity, internal heat gains, wind speed on the building thermal dynamics is assumed to be negligible compared to the influence of the ambient temperature, the solar radiation power, and HVAC output power.

Note that these assumptions are commonly made in the literature [23]-[25], [28]. The thermal energy from solar irradiance through windows can be calculated as [23]

$$
Q_{k, i}^{\mathrm{s}}=\Phi_{i}^{\mathrm{s}} A_{k}
$$

where $\Phi_{i}^{\mathrm{s}}$ is the solar irradiance and $A_{k}$ is the effective window area of house $k$.

Assume that all energy flux by solar radiation through windows is absorbed by the heat accumulating layer in the inner walls and the indoor air. We define $p_{k}^{\mathrm{s}}$ as the fraction of solar radiation entering the inner walls and floor of house $k$, then the rest of the solar energy is absorbed by the indoor air, i.e., we have

$$
\begin{aligned}
Q_{k, i}^{s, \text { wall }} & =p_{k}^{\mathrm{s}} Q_{k, i}^{\mathrm{s}} \\
Q_{k, i}^{s, \text { air }} & =\left(1-p_{k}^{\mathrm{s}}\right) Q_{k, i}^{\mathrm{s}}
\end{aligned}
$$

where $Q_{k, i}^{s, \text { wall }}$ and $Q_{k, i}^{s, i}$ denote these two energy fractions, respectively. In general, heat transfer occurs when there is a temperature difference between two spaces. Thermal energy is transferred from a higher temperature space toward a lower temperature space due to conduction, convection, and radiation [23]. Based on heat transfer mechanisms, we construct the energy balance equations, which consequently result in a third 
order linear model for thermal dynamics of house $k$ as follows [23]:

$$
\begin{aligned}
C_{k}^{\mathrm{m}} \frac{d T_{k}^{\mathrm{m}}}{d t}= & \frac{1}{R_{k}^{\mathrm{m}}}\left(T_{k}-T_{k}^{\mathrm{m}}\right)+A_{k} p_{k}^{\mathrm{s}} \Phi_{k}^{\mathrm{s}} \\
C_{k}^{\mathrm{e}} \frac{d T_{k}^{\mathrm{e}}}{d t}= & \frac{1}{R_{k}^{\mathrm{e}}}\left(T_{k}-T_{k}^{\mathrm{e}}\right)+\frac{1}{R_{k}^{\mathrm{ea}}}\left(T^{\mathrm{a}}-T_{k}^{\mathrm{e}}\right) \\
C_{k} \frac{d T_{k}}{d t}= & \frac{1}{R_{k}^{\mathrm{a}}}\left(T^{\mathrm{a}}-T_{k}\right)+\frac{1}{R_{k}^{\mathrm{m}}}\left(T_{k}^{\mathrm{m}}-T_{k}\right) \\
& +\frac{1}{R_{k}^{\mathrm{e}}}\left(T_{k}^{\mathrm{e}}-T_{k}\right)+A_{k}\left(1-p_{k}^{\mathrm{s}}\right) \Phi_{k}^{\mathrm{s}}+\sigma_{k} P_{k}^{\text {hvac,out }} .
\end{aligned}
$$

where $\sigma_{k}=1$ corresponds to the winter time and $\sigma_{k}=-1$ for the summer time. Other parameters are defined as

- $R_{k}^{\mathrm{a}}$ is the resistance between room air and the ambient $\left({ }^{\circ} \mathrm{C} / \mathrm{kW}\right)$.

- $R_{k}^{\mathrm{m}}$ is the thermal resistance between room air and the the thermal accumulating layer in the inner walls and floor $\left({ }^{\circ} \mathrm{C} / \mathrm{kW}\right)$.

- $R_{k}^{\mathrm{e}}$ is the thermal resistance between room air and the the house envelope $\left({ }^{\circ} \mathrm{C} / \mathrm{kW}\right)$.

- $R_{k}^{\text {ea }}$ is the thermal resistance between the house envelope and the the ambient $\left({ }^{\circ} \mathrm{C} / \mathrm{kW}\right)$.

- $C_{k}$ is total thermal capacitance of the indoor air $\left(\mathrm{kWh} /{ }^{\circ} \mathrm{C}\right)$.

- $C_{k}^{\mathrm{m}}$ is the total thermal capacitance of the inner walls $\left(\mathrm{kWh} /{ }^{\circ} \mathrm{C}\right)$.

- $C_{k}^{\mathrm{e}}$ is the total thermal capacitance of the house envelope $\left(\mathrm{kWh} /{ }^{\circ} \mathrm{C}\right)$.

The equivalent thermal parameters $R_{k}^{\mathrm{a}}, R_{k}^{\mathrm{m}}, R_{k}^{\mathrm{e}}, R_{k}^{\mathrm{ea}}, C_{k}$, $C_{k}^{\mathrm{m}}, C_{k}^{\mathrm{e}}$, and $p_{k}^{\mathrm{s}}$ are assumed to be constant, which can be estimated by using the Maximum Likelihood (ML) method based on measured data [23]-[25], [27]. Therefore, the thermal dynamics of $k$-th house can be rewritten in the deterministic linear state space model in continuous time as

$$
\begin{aligned}
\frac{d T_{k}}{d t} & =A T_{k}+B U_{k} \\
T_{k}^{r} & =C T_{k}
\end{aligned}
$$

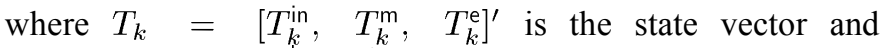
$\mathrm{U}_{k}=\left[T^{\mathrm{a}}, \Phi^{s}, \sigma_{k} P_{k}^{\text {hvac out }}\right]^{\prime}$ is the input vector to the system. The output of interest is $T_{k}^{r}=T_{k}^{\text {in }}$ because we are interested in the indoor temperature, which directly impacts user climate comfort. Matrix $A$ represents the dynamic behavior of the system, and matrix $B$ captures the impact of input elements (ambient temperature, solar radiation, and HVAC power) on the system behavior. The matrices in the state space model (5) are given as follows:

$$
A=\left[\begin{array}{ccc}
a_{11} & a_{12} & a_{13} \\
a_{21} & a_{22} & 0 \\
a_{31} & 0 & a_{33}
\end{array}\right]
$$

where the underlying coefficients are defined as

$$
\begin{aligned}
& a_{11}=\frac{-1}{C_{k}}\left(\frac{1}{R_{k}^{\mathrm{a}}}+\frac{1}{R_{k}^{\mathrm{m}}}+\frac{1}{R_{k}^{\mathrm{e}}}\right) \\
& a_{12}=\frac{1}{R_{k}^{\mathrm{m}} C_{k}} ; \quad a_{13}=\frac{1}{R_{k}^{\mathrm{e}} C_{k}} \\
& a_{21}=\frac{1}{R_{k}^{\mathrm{m}} C_{k}^{\mathrm{m}}} ; \quad a_{22}=-\frac{1}{R_{k}^{\mathrm{m}} C_{k}^{\mathrm{m}}}
\end{aligned}
$$

$$
\begin{aligned}
a_{31} & =\frac{1}{R_{k}^{\mathrm{e} C} C_{k}^{\mathrm{e}}} ; \quad a_{33}=-\frac{1}{C_{k}^{\mathrm{e}}}\left(\frac{1}{R_{k}^{\mathrm{ea}}}+\frac{1}{R_{k}^{\mathrm{e}}}\right) \\
B & =\left[\begin{array}{ccc}
\frac{1}{R_{k}^{\mathrm{a}} C_{k}} & \frac{A_{k}\left(1-p_{k}^{\mathrm{s}}\right)}{C_{k}} & \frac{1}{C_{k}} \\
0 & \frac{A_{k} p_{k}^{\mathrm{s}}}{C_{k}^{m}} & 0 \\
\frac{1}{R_{k}^{\mathrm{ea} C} C_{k}^{\mathrm{e}}} & 0 & 0
\end{array}\right] \\
C & =\left[\begin{array}{lll}
1 & 0 & 0
\end{array}\right] .
\end{aligned}
$$

The state space model in continuous time (5) can be transformed into the equivalent discrete time model by using Euler discretization (i.e., zero-order hold) with a sampling time of $T_{s}$ [23], [27] as

$$
\begin{aligned}
T_{k, i+1} & =A_{d} T_{k, i}+B_{d} U_{k, i} \\
T_{k, i}^{r} & =C_{d} T_{k, i}
\end{aligned}
$$

where

$$
\begin{aligned}
& A_{d}=\exp \left(A T_{s}\right)=1+A T_{s}+\frac{A^{2} T_{s}^{2}}{2}+\cdots \\
& B_{d}=\int_{0}^{T_{s}} \exp (A \tau) d \tau B ; \quad C_{d}=C
\end{aligned}
$$

This discrete time thermal dynamic model will be used in the following energy scheduling problem formulation.

\section{Problem Formulation}

We present the joint EV charging and home energy management problem for cost minimization in this section. First, the total electricity power imported from the grid at time slot $i$ can be written as

$$
P_{i}^{\text {grid }}=\sum_{k=1}^{M} P_{k, i}^{\mathrm{hvac}}+\sum_{k=1}^{M} \sum_{j=1}^{J_{k}}\left[P_{k, j, i}^{\mathrm{ev}, c}-P_{k, j, i}^{\mathrm{ev}, d}\right],
$$

which is equal to EV charging power plus power usage of the HVAC system minus the EV discharging power summed over all households. The aggregator aims to minimize the total electricity cost and user discomfort during a scheduling horizon. We assume that there is no electricity losses in the transmission lines among EVs and HVAC systems. This assumption is reasonable since the transmission lines for energy exchange in a community are relatively short. Moreover, EV charging is assumed to be continuously controllable in our control model. The objective and constraints of the underlying optimization problem are described in the following.

\section{A. Objective Function}

The objective function consists of two parts. The first part is the total electricity cost which can be expressed as

$$
J_{\text {elec }}=\sum_{i=1}^{N} \sum_{k=1}^{M} P_{i}^{\text {grid }} e_{i} \Delta T .
$$

Substitute the result of (8) into (9), we have

$$
J_{\text {elec }}=\sum_{i=1}^{N}\left(\sum_{k=1}^{M} P_{k, i}^{\mathrm{hvac}}+\sum_{k=1}^{M} \sum_{j=1}^{J_{k}}\left[P_{k, j, i}^{\mathrm{ev}, c}-P_{k, j, i}^{\mathrm{ev}, d}\right]\right) e_{i} \Delta T .
$$


The second part of the objective function is the discomfort cost. It is assumed that each household $k$ will inform its preferred temperature $T_{k, i}^{\mathrm{d}}$ for each time slot $i$ when the house is occupied and a maximum acceptable deviation $\delta_{k}$ to the aggregator. Then, the aggregator controls the power supplied to HVAC system so that the temperature lies within the acceptable range $\left[T_{k, i}^{\mathrm{d}}-\right.$ $\left.\delta_{k}, T_{k, i}^{\mathrm{d}}+\delta_{k}\right]$ in each occupied house $k$. The closer to the desired temperature, the more comfortable users would be. There is no indoor temperature constraint for the period when the house is unoccupied. The power supplied to an HVAC system at time slot $i$ will decide the temperature at time slot $i+1$, which consequently affects user comfort at time slot $i+1$ but time slot $i$. Therefore, we define the discomfort cost function as

$$
J_{\text {discomfort }}=\sum_{i=1}^{N} \sum_{k=1}^{M} w_{k} a_{k, i+1}\left|T_{k, i+1}^{\mathrm{in}}-T_{k, i+1}^{\mathrm{d}}\right|,
$$

where $a_{k, i+1}$ represents the occupancy status of house $k$ at time slot $i+1$. If $a_{k, i+1}=0$, the discomfort cost for house $k$ at time slot $i+1$ is equal to zero regardless of the indoor temperature at that time slot because the house is not occupied. The weighting factor $w_{k}$ can be viewed as the price (\$) that aggregator has to pay household $k$ when the temperature in house $k$ deviates 1 ${ }^{\circ} \mathrm{C}$ from the desired temperature in each time slot. The value of $w_{k}$ will influence the optimal solution of the underlying optimization problem. The objective function, which is the sum of electricity cost and the discomfort cost, can be written as follows:

$$
\begin{array}{r}
J_{\mathrm{tot}}=\sum_{i=1}^{N}\left(\sum_{k=1}^{M} P_{k, i}^{\mathrm{hvac}}+\sum_{k=1}^{M} \sum_{j=1}^{J_{k}}\left[P_{k, j, i}^{\mathrm{ev}, c}-P_{k, j, i}^{\mathrm{ev}, d}\right]\right) e_{i} \Delta T \\
+\sum_{i=1}^{N} \sum_{k=1}^{M} w_{k} a_{k, i+1}\left|T_{k, i+1}^{\mathrm{in}}-T_{k, i+1}^{\mathrm{d}}\right|
\end{array}
$$

We are now ready to describe all constraints for the considered optimization problem.

\section{B. Thermal Constraints}

1) Thermal Dynamics Model: The thermal dynamics model for a residential house has been presented in Section III. It can be seen from (7), for each household, the indoor temperature at the next time slot is determined by the current indoor temperature $\left(T_{k, i}^{\mathrm{in}}\right)$, the current outdoor temperate $\left(T_{i}^{\mathrm{a}}\right)$, the solar radiation power $\left(Q_{k, i}^{s}\right)$, and the output power of the HVAC system $\left(P_{k, i}^{\text {hvac,out }}\right)$ at the current time slot. The output power is related to the power supplied to the HVAC system as $P_{k, i}^{\text {hvac,out }}=\eta_{k} P_{k, i}^{\text {hvac }}$ where $\eta_{k}$ is the performance coefficient of the HVAC system in household $k$. The discrete time thermal dynamic model (7) represents one constraint of the considering optimization problem $\left(T_{k, i}^{r}=T_{k, i}^{\text {in }}\right)$.

2) Temperature Constraints: Each household informs its desired temperature to the aggregator. Then, the aggregator controls power supplied to the HVAC system in the house at each time slot to keep the indoor temperature as close as possible to the desired temperature. The indoor temperature requirement for each house is expressed as

$$
a_{k, i}\left|T_{k, i}^{\mathrm{in}}-T_{k, i}^{\mathrm{d}}\right| \leq \delta_{k},
$$

for $k=1,2, \ldots, M$ and $i=2, \ldots, N+1$. There is no temperature requirement when a house is not occupied. Note that the power provided to an HVAC system in the current time slot will affect the indoor temperature in the next time slot, so the temperature constraint is only applied from the second time slot.

3) HVAC Power Constraints: The power supplied to an HVAC system cannot be negative and it cannot take values greater than the heater/AC's power rating. Therefore, we have

$$
0 \leq P_{k, i}^{\text {hvac }} \leq P_{k}^{\text {hvac,max }},
$$

for $k=1,2, \ldots, M$ and $i=1, \ldots, N$.

Remark 1: In our proposed system model, each household needs to report its desired temperature at each time slot during which the household is occupied as well as the level of discomfort (i.e., parameters $\delta_{k}$ ) to the aggregator to determine the optimal control solution. In practice, if a particular user does not wish to report its desired temperature in the occupied time slots to the aggregator then the aggregator can simply choose a typical temperature value for this household to calculate the optimal solution.

\section{SOC and Charging Power Constraints}

For EVs, we need to model the characteristics and the travel patterns for each EV. In particular, we are interested in the battery capacity $(\mathrm{kW})$, the travel efficiency $(\mathrm{kWh} / \mathrm{mile})$, and the charging type. These properties can be retrieved from the manufacturer's website. The travel pattern of each EV can be described by the number of trips per day, the starting and ending times, and the travel distance of each trip. A trip is defined as the time period between the instants when the EV leaves and arrives home. This information is related to user traveling schedule, which can be sent by users to the aggregator before the operating day. In Section V, we use the real-world travel pattern data from the 2009 National Household Travel Survey [30] to build travel patterns used to obtain numerical results.

1) SOC Dynamics: Assume that each EV $j$ of house $k$ can take several trips during the optimization period (e.g., one day). Let $t_{k, j, l}^{(1)}$ and $t_{k, j, l}^{(2)}$ be the time slots when EV $j$ of house $k$ leaves and arrives home for trip $l$, respectively. Then, we have following constraints

$$
\begin{aligned}
S O C_{k, j, i+1} & =S O C_{k, j, i}+\frac{\eta_{k, j}^{\mathrm{c}} P_{k, j, i}^{\mathrm{ev}, c} \Delta T}{E_{k, j}^{\mathrm{cap}}}-\frac{P_{k, j, i}^{\mathrm{ev}, d} \Delta T}{\eta_{k, j}^{\mathrm{d}} E_{k, j}^{\mathrm{cap}}}, \\
& \text { if } i \notin\left[t_{k, j, l}^{(1)}, t_{k, j, l}^{(2)}\right), \quad \forall k, i, j, l \\
S O C_{k, j, i+\Lambda_{k, j, l}} & =S O C_{k, j, i}-\frac{d_{k, j, l} * m_{k}}{E_{k, j}^{\mathrm{cap}}}, \\
\text { if } i & =t_{k, j, l}^{(1)}, \quad \forall k, i, j, l \\
S O C_{k, j, t_{k, j, l}^{(2)}} \leq S O C_{k, j, i} \leq S O C_{k, j, t_{k, j, l}^{(1)}} & \\
& \text { if } i \in\left[t_{k, j, l}^{(1)}, t_{k, j, l}^{(2)}\right], \quad \forall k, i, j, l .
\end{aligned}
$$

Here, the SOC for EV $j$ of house $k$ changes according to the charging and discharging powers when it parks at home (15) and the difference of SOCs at leaving and returning home instants accounts for the energy usage in driving (16). Equation (17) ensures that the SOC level is non-increasing when an EV travels.

2) SOC Constraints: To maintain long lifetime of battery, an EV should maintain its battery level within a certain range that 
is recommended by its manufacturer [31]. Therefore, we impose the following constraints

$$
S O C_{k, j}^{\min } \leq S O C_{k, j, i} \leq S O C_{k, j}^{\max }, \quad \forall k, j, i
$$

where $S O C_{k, j}^{\min }$ and $S O C_{k, j}^{\max }$ denote the minimum and maximum recommended SOCs for EV $j$ of house $k$.

3) Charging and Discharging Constraints: We assume that an EV is only charged or discharged when it is parked at home. Moreover, EVs are connected to home chargers as soon as they arrive home. Therefore, constraints on charging and discharging power applied to only time slots when an EV is parked at home as

$$
\begin{aligned}
& 0 \leq P_{k, j, i}^{\mathrm{ev}, c} \leq b_{k, j, i} P_{k, j}^{\mathrm{ev}, c, \text { max }} \\
& 0 \leq P_{k, j, i}^{\mathrm{ev}, d} \leq b_{k, j, i} P_{k, j}^{\mathrm{ev}, d, \text { max }} .
\end{aligned}
$$

where $b_{k, j, i}$ represents the availability of EV $j$ of house $k$ at home during time slot $i, P_{k, j}^{\mathrm{ev}, c, \max }$ and $P_{k, j}^{\mathrm{ev}, d, \max }$ denote the maximum charging and discharging limits, respectively. From these constraints, the charging and discharging powers for each $\mathrm{EV} j$ of household $k$ (i.e., $P_{k, j, i}^{\mathrm{ev}, c}$ and $P_{k, j, i}^{\mathrm{ev}, d}$ ) are equal zero if the $\mathrm{EV}$ is not at home (i.e., as $b_{k, j, i}=0$ ).

\section{Grid Constraints}

If it is not allowed to sell EV discharging energy back to the main grid, the energy imported from the grid in each time slot must be non-negative and it must be upper-bounded by some predetermined limit. Hence, we have

$$
0 \leq P_{i}^{\text {grid }} \leq P_{i}^{\max }
$$

or

$$
0 \leq \sum_{k=1}^{M} P_{k, i}^{\mathrm{hvac}}+\sum_{k=1}^{M} \sum_{j=1}^{J_{k}}\left[P_{k, j, i}^{\mathrm{ev}, c}-P_{k, j, i}^{\mathrm{ev}, d}\right] \leq P_{i}^{\max },
$$

where $P_{i}^{\max }$ is the maximum power that can be imported from the grid, which can be a contracted amount between the aggregator and the grid, or a particular parameter capturing grid conditions over time. In contrast, if the selling EV discharging energy service is allowed and the maximum power that can be sold back to the main grid is equal to $P_{i}^{\max }$. Then, we have the following constraint

$$
-P_{i}^{\max } \leq \sum_{k=1}^{M} P_{k, i}^{\mathrm{hvac}}+\sum_{k=1}^{M} \sum_{j=1}^{J_{k}}\left[P_{k, j, i}^{\mathrm{ev}, c}-P_{k, j, i}^{\mathrm{ev}, d}\right] \leq P_{i}^{\max } .
$$

For simplicity, we assume that the selling back electricity price is equal to the buying electricity price. In summary, we can formulate the EV charging and HVAC scheduling to minimize the cost function $J_{\text {tot }}$ given in (12) as

$$
\begin{aligned}
\min & \sum_{i=1}^{N}\left(\sum_{k=1}^{M} P_{k, i}^{\mathrm{hvac}}+\sum_{k=1}^{M} \sum_{j=1}^{J_{k}}\left[P_{k, j, i}^{\mathrm{ev}, c}-P_{k, j, i}^{\mathrm{ev}, d}\right]\right) e_{i} \Delta T \\
& +\sum_{i=1}^{N} \sum_{k=1}^{M} w_{k} a_{k, i+1}\left|T_{k, i+1}^{\mathrm{in}}-T_{k, i+1}^{\mathrm{d}}\right|
\end{aligned}
$$

subject to

$$
\begin{array}{r}
\text { constraints(7), (13)-(19) } \\
\text { constraints (21), if no V2G } \\
\text { constraints(22), if V2G (23) }
\end{array}
$$

where the optimization variables are $P_{k, j, i}^{\mathrm{ev}, c}, P_{k, i}^{\text {hvac }}$, and $P_{k, j, i}^{\mathrm{ev}, d}$. Despite the absolute term in the objective function of our model, this optimization problem can be transformed into an equivalent linear program by introducing some auxiliary variables [32]. Thus, the aggregator can easily calculate and implement its optimal solution upon collecting all required information.

It can be observed that we do not impose constraints in the optimization problem (23) to prevent any EV $j$ of house $k$ from charging and discharging simultaneously at any time slot $i$ (i.e., $P_{k, j, i}^{\mathrm{ev}, c}$ and $P_{k, j, i}^{\mathrm{ev}, d}$ for any EV $j$ of house $k$ are both positive at the same time slot $i$ ). In fact, this is not needed since the optimal solution of (23) always satisfies these hidden constraints.

\section{E. Extensions to Consider Modeling Uncertainties and Other Residential Loads}

In the above formulation, we have assumed that all modeling parameters such as outdoor temperature, household occupancy pattern, and EV travel pattern are known without errors and the thermal dynamics model is perfect. In practice, they have to be estimated with potential errors. We can employ the Model Predictive Control (MPC) technique to tackle these estimation uncertainties [33], which can be implemented as follows. The MPC controller solves the minimization problem (23) for the prediction horizon $N_{0}$ from current time slot $t$ to time slot $t+$ $N_{0}$ with assumption that estimated parameters are certain ones (i.e., no estimation errors). The uncertainties are compensated by refinement and update of the prediction at each time step. The sequences of control variables such as power consumption of HVAC and EVs are calculated for the whole prediction horizon; however, the controller applies only the control action for the first time slot. The MPC controller repeats the process at next time step, solving new optimization with the most updated data for the new time horizon shifted one step forward.

We have only considered EVs and HVAC systems in our proposed optimization framework so far. However, integration of other types of residential loads into this framework is possible. Moreover, extension of our system model to consider distributed renewable energy sources is also possible. Here, the MPC technique can be employed again to tackle the uncertainty due to the intermittent nature of the underlying renewable sources (e.g., wind or solar energy).

\section{Numerical Results}

We present numerical results to illustrate the desirable performance of the proposed framework. We assume that the outdoor temperature and solar irradiance can be predicted perfectly. The temperature data is taken from Weather Underground website [34], and the solar irradiance data is taken from the Renewable Resource Data Center (RReDC) website [35]. Solar radiation power contributes to increase the indoor house temperature; therefore, it results in more cooling energy needed in the summer and less heating energy needed in the winter. Moreover, the solar irradiance is low during the winter months and high during summer months as we can observe in Fig. 2(d). Hence, 
the decreasing amount of heating energy required in the winter is relatively small compared to the increasing amount of cooling energy needed in the summer.

For the electricity price data, we use day-ahead pricing data retrieved from PJM [36]. We will first evaluate the performance of our control scheme for a single-house scenario. Then, we investigate the benefits of applying the control strategy in the multiple-house setting. Results for the single-house scenario are presented to reveal insights into the interaction among the HVAC system, EV, pricing, and temperature patterns.

When solving the joint scheduling optimization for EVs and HVAC systems we set $\sigma_{k}=-1$ and $\sigma_{k}=1$ for all $k$ corresponding to the summer and winter time, respectively. The optimization period is one day with 24 time slots each of which is one hour $(N=24, \Delta T=1)$. Fig. 2(a) shows the dayahead electricity prices of a typical summer and winter weekdays, which are used to obtain numerical results. Three different temperature profiles for summer days (very hot, hot, mild) and winter days (very cold, cold, mild) are considered to represent the diversity of weather conditions, as shown in Fig. 2(b) and (c), respectively. The average hourly solar irradiance profiles for the summer and winter cases used in the simulation are shown in Fig. 2(d). We use CVX [37] to solve the proposed optimization problem.

\section{A. Single-House Scenario}

We analyze the performance due to our proposed optimal scheme to the single-house scenario. For simplicity, we assume that the considering residential house has only one EV and one HVAC where the varying ownership aspect will be captured later in Section V-B for the multiple-house case. The housing thermal parameters including $R_{k}^{\mathrm{a}}, R_{k}^{\mathrm{m}}, R_{k}^{\mathrm{e}}, R_{k}^{\mathrm{ea}}, C_{k}, C_{k}^{\mathrm{m}}$, and $C_{k}^{\mathrm{e}}$ are taken from [23]. We assume that the house is equipped with a heat pump which can be operated in both heating and cooling modes. The parameters of the heat pump are set as follows: power rating $P_{k}^{\text {hvac, max }}=4 \mathrm{~kW}$ and HVAC coefficient of performance (COP) $\eta_{k}=3$. We consider Nissan Leaf EVs whose specifications are obtained from [31] with the following parameter setting: battery capacity of $24 \mathrm{kWh}$; maximum charging and discharging power $\left(P_{k}^{\mathrm{ev}, c, \max }\right.$ and $\left.P_{k}^{\mathrm{ev}, d, \max }\right)$ are set equally to $6 \mathrm{~kW}$ [38]; charging and discharging efficiency factors $\left(\eta_{c}\right.$ and $\left.\eta_{d}\right)$ are both set equal to 0.9 ; travel efficiency is $0.316 \mathrm{kWh} / \mathrm{mile}$; and $S O C_{k, j}^{\max }=0.9, S O C_{k, j}^{\min }=0.2$. The initial SOC of the EV is set equal to 0.5. To obtain numerical results for the single-house scenario, we simply set $M=1$ in all related constraints and quantities.

We assume that the EV's owner leaves home at 8 A.M. and comes back at 5 P.M. Driving distance is assumed to be 32 miles, which is the average daily travel distance in the U.S. [17], [30], [41]. This is a typical driving pattern [16] in the U.S., which is used to obtain the numerical results in several scenarios below. However, other different driving patterns are also examined where we will investigate the impacts of varying departure time, arrival time, and travel distance on the optimal solution. In addition to the above parameter settings for HVAC system and EV, the power limit $P_{i}^{\max }$ is set equal to $25 \mathrm{~kW}$. The desired indoor temperature in summer days and winter days are $23{ }^{\circ} \mathrm{C}$ and $21^{\circ} \mathrm{C}$, respectively. Initial temperature at 0 A.M. is assumed

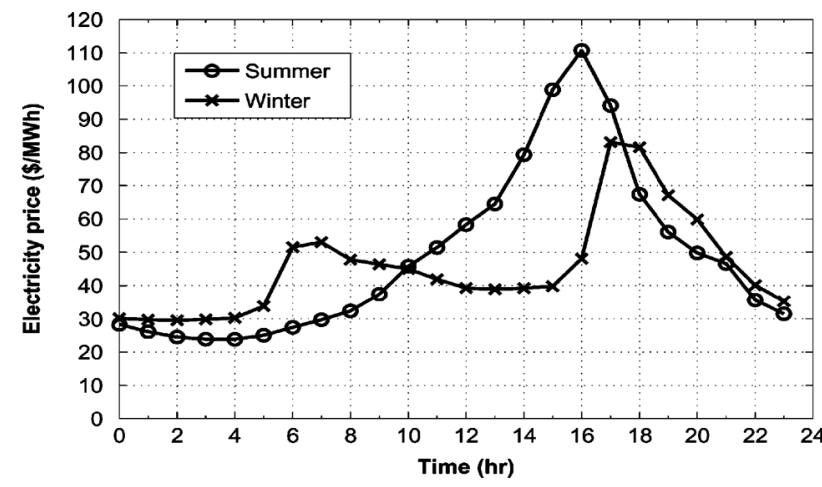

(a)

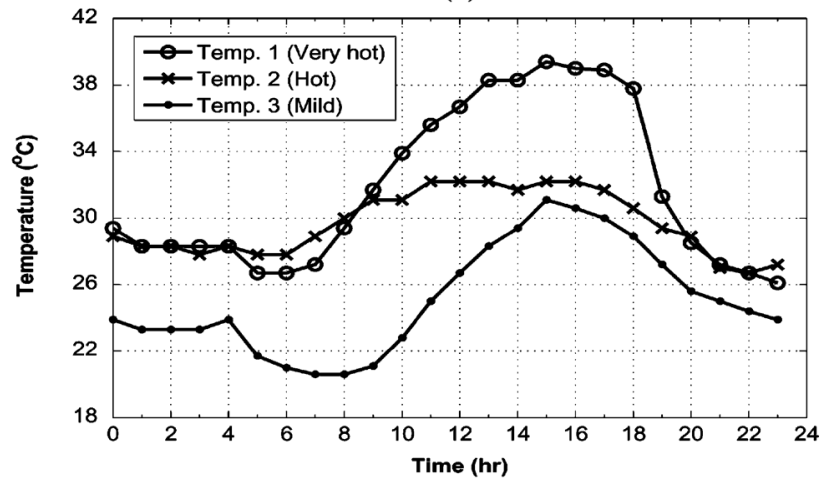

(b)

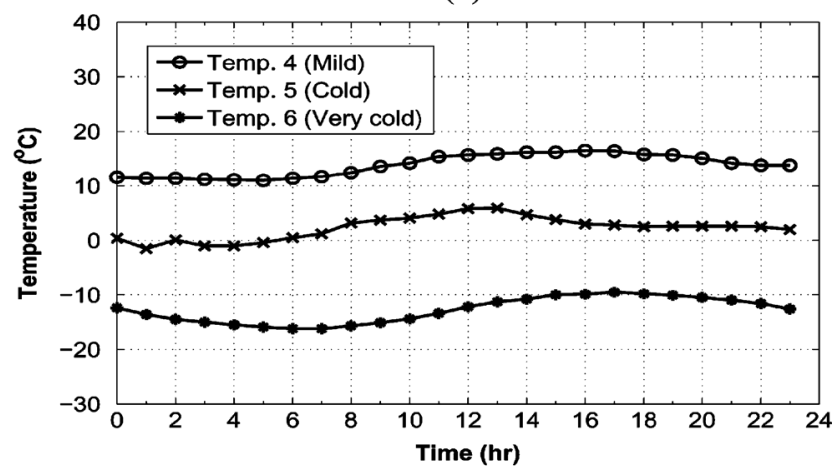

(c)

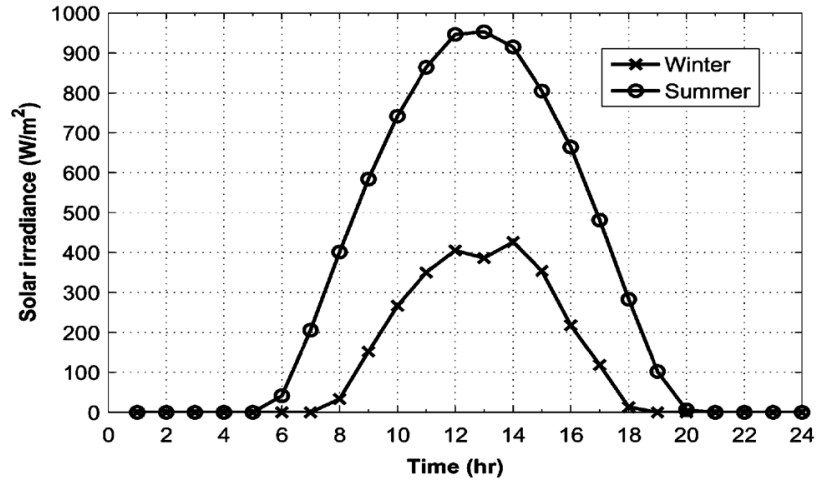

(d)

Fig. 2. Electricity price and weather profiles. (a) Day-ahead electricity price. (b) Summer temperature profiles. (c) Winter temperature profiles. (d) Solar irradiance.

to be equal to the desired indoor temperature. Assuming that the considered house is occupied all day, so the desired indoor temperature is equal to $23{ }^{\circ} \mathrm{C}$ (summer) or $21{ }^{\circ} \mathrm{C}$ (winter) at every time slot. 


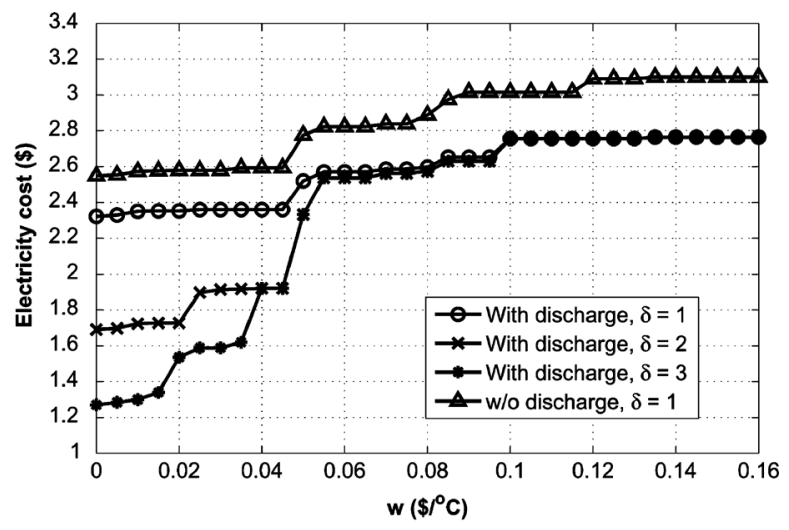

(a)

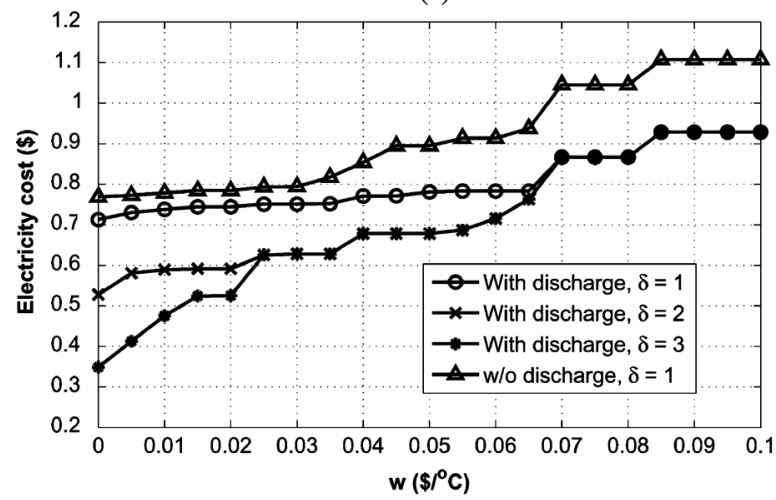

(b)

Fig. 3. Impacts of parameters $w$ and $\delta$ on electricity cost (No V2G). (a) Summer (very hot). (b) Winter (cold).

Fig. 3(a) and (b) show the impacts of parameters $\delta$ and $w$ on the total electricity cost in a summer day and a winter day, respectively. The weighting factor $w$ is varied in a certain interval, and $\delta$ takes one of three values: $1{ }^{\circ} \mathrm{C}, 2{ }^{\circ} \mathrm{C}$, and $3{ }^{\circ} \mathrm{C}$. To enforce the stricter user comfort requirement, we would choose a higher value for $w$ and a smaller value for $\delta$. These figures show that the electricity cost increases as $w$ increases. This is intuitive since the cost of electricity increases with stricter user comfort requirement. Moreover, for small values of $w$ (i.e., $w<0.04 \$ /{ }^{\circ} \mathrm{C}$ for the summer day, and $w<0.025 \$ /{ }^{\circ} \mathrm{C}$ for the winter day), the electricity cost decreases as $\delta$ increases. This is because small values of $w$ allow the indoor temperature to deviate more significantly from the preferred value to save electricity cost, especially for large values of $\delta$. However, as $w$ becomes sufficiently large (i.e., $w>0.1 \$ /{ }^{\circ} \mathrm{C}$ for the summer day, and $w>0.07 \$ /{ }^{\circ} \mathrm{C}$ for the winter day), the electricity costs corresponding to the three different values of $\delta$ are the same. This is because sufficiently high values of penalty value $w$ results in the temperature being close to the desired value for the whole day. These figures also show that the electricity cost due to optimal control without discharging is much higher than that exploiting EV discharging capability. This result confirms the great benefits of exploiting interactions among EVs and between EVs and HVAC systems. To obtain numerical results for the optimal control without discharging, we simply set maximum discharging power to zero in our problem formulation.

Due to the space constraint, we consider only the summer case to present other numerical results in the following. Fig. 4(a)

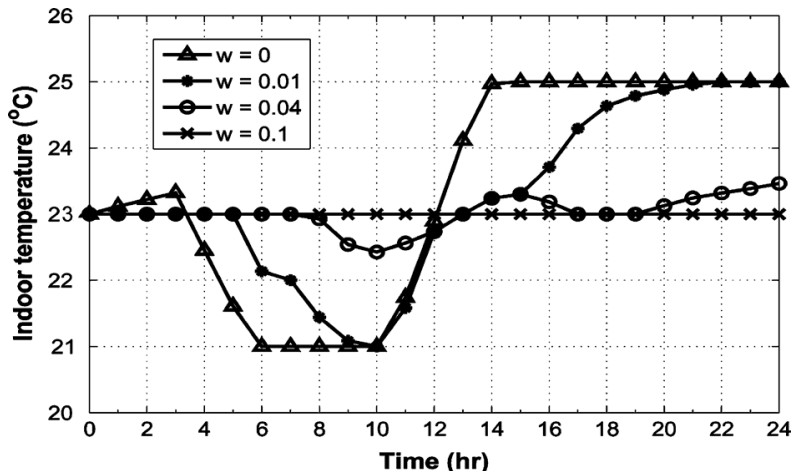

(a)

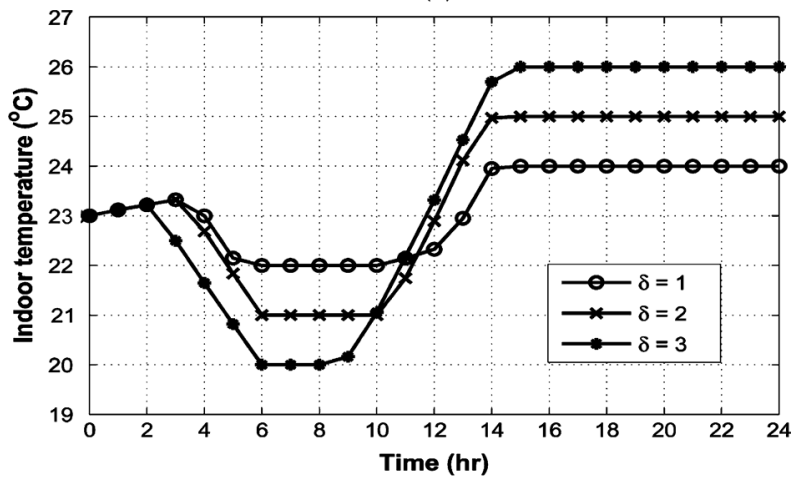

(b)

Fig. 4. Impacts of parameters $w$ and $\delta$ on indoor temperature (No V2G). (a) Impact of $w$ on indoor temperature $\left(\delta=2{ }^{\circ} \mathrm{C}\right)$. (b) Impact of $\delta$ on indoor temperature $\left(w=0.02 \$ /{ }^{\circ} \mathrm{C}\right)$.

illustrates the indoor temperature variation over time for $\delta=$ $2{ }^{\circ} \mathrm{C}$ and different values of $w$. This figure confirms our observation from Fig. 3(a) where higher values of $w$ reduce the fluctuation of indoor temperature around its preferred value. In Fig. 4(b) we plot the indoor temperature over time for a fixed $w$ and different values of $\delta$. This figure again indicates that the indoor temperature oscillates more around the preferred value as $\delta$ increases.

Fig. 5(a) shows the power imported from the grid under our control scheme exploiting EV discharging capability compared to optimal solution without discharging and the uncontrolled scheme in the summer day. For the uncontrolled scheme, EV charging occurs at midnight when EV is plugged, regardless of electricity price. The charging terminates when the EV meets the energy consumption requirement for the day. For fair comparison, the energy charged to $\mathrm{EV}$ is chosen so that the remaining energy in the EV battery at the end of the day equal to the energy in the controlled case. In addition, for the uncontrolled case, the $\mathrm{AC}$ is controlled by a thermostat to keep the indoor temperature equal to the desired temperature at every time slots. It can be observed that our optimal scheme with discharging reduces the significantly load during peak hours (from 2 P.M. to 9 P.M.) when the electricity price is very high [cf. Fig. 2(a)]. The negative value of imported power in the $\mathrm{V} 2 \mathrm{G}$ case represents the power selling back to the grid. To supply energy for the HVAC system, the EV discharges its remaining battery right after it arrives home as indicated in Figs. 5(b), (c). Moreover, during high-price hours the amount 


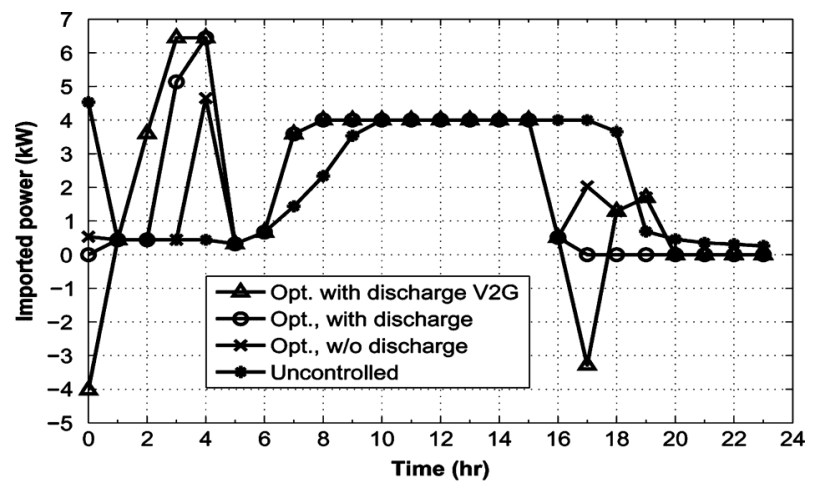

(a)

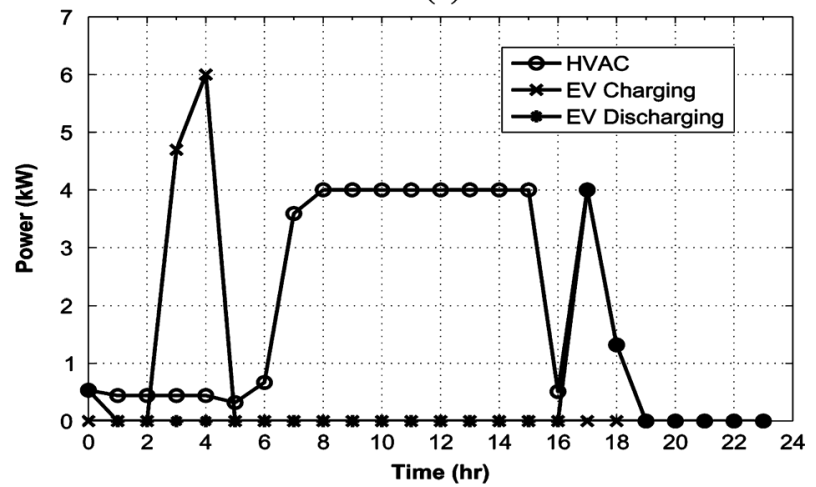

(b)

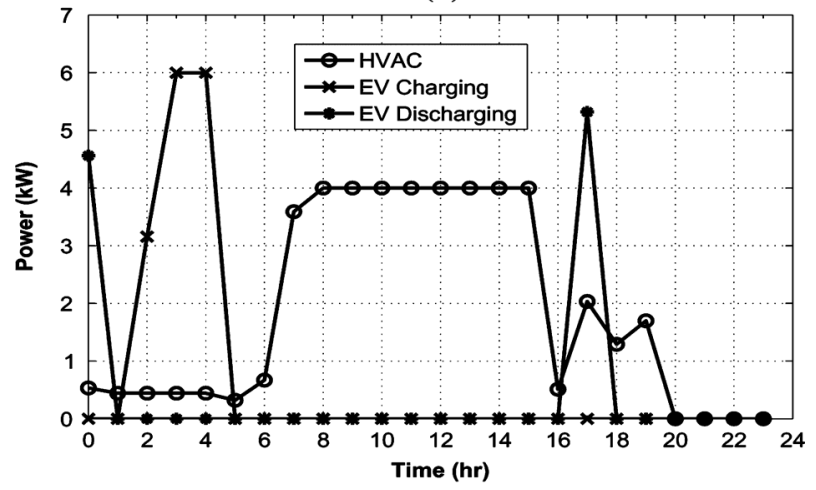

(c)

Fig. 5. Imported power and power consumption (very hot day, $w=0.01 \$ /{ }^{\circ} \mathrm{C}$, $\delta=2{ }^{\circ} \mathrm{C}$ ). (a) Imported power. (b) Power consumption, charging/discharging (No V2G). (c) Power consumption, charging/discharging (V2G).

of the EV discharging power for the V2G case in Fig. 5(c) is much higher than the amount of the $\mathrm{EV}$ discharging power without V2G service in Fig. 5(b). EV charging occurs at time slots when electricity prices are low (from 3 A.M. to 4 A.M.).

In Figs. 6(a), (b), we present the impacts of different temperature profiles on electricity cost saving compared to the uncontrolled scheme in a summer day. It can be observed that the cost saving decreases with increasing $w$ since larger values of $w$ reduces the flexibility in controlling HVAC consumption. Also, the absolute cost saving in dollars is larger for a hot or very hot day than that for a mild day. This is because more EV discharging energy to the HVAC system would be expected in a hot or very hot day, which translates into more significant cost saving. Fig. 6(b) shows that for a small value of $w$ (e.g., less than $0.04 \$ /{ }^{\circ} \mathrm{C}$ ), the cost saving of more than $25 \%$ can be achieved

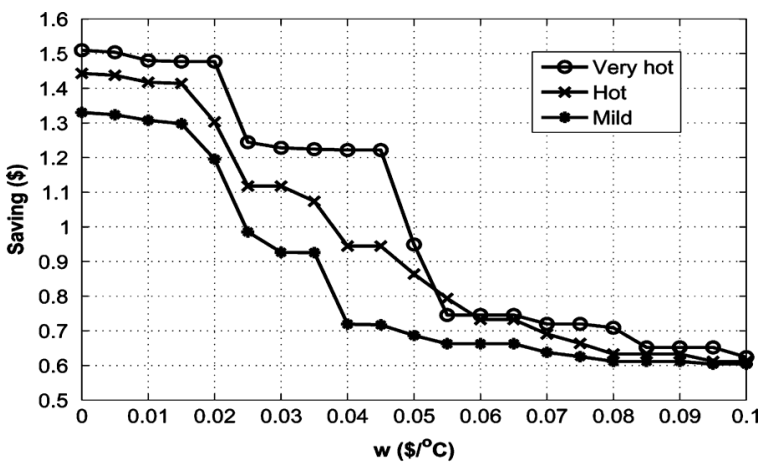

(a)

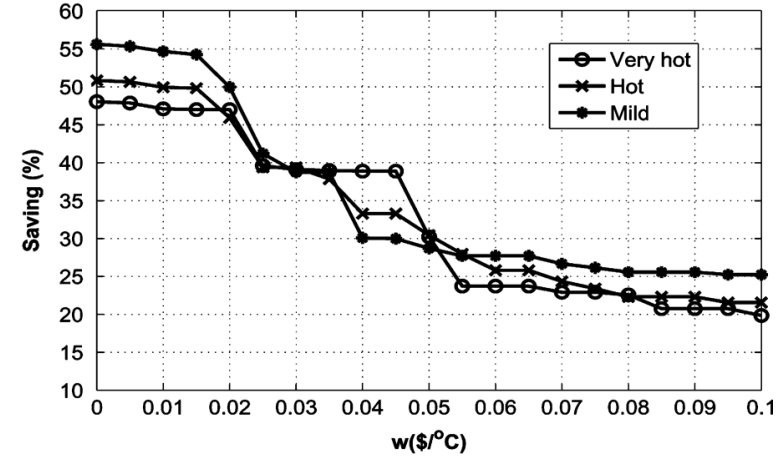

(b)

Fig. 6. Impact of temperature profiles and $w$ on cost saving (No V2G, $\delta=$ $2{ }^{\circ} \mathrm{C}$ ). (a) Absolute cost saving. (b) Relative cost saving.

in a mild or hot day where the relative cost saving is calculated as

Saving $(\%)$

$$
=\frac{\text { Cost of uncontrolled case }- \text { Optimal cost }}{\text { Cost of uncontrolled case }} 100 .
$$

We illustrate the absolute and relative cost saving compared to the uncontrolled scheme versus users' arrival and departure times for different summer temperature profiles in Figs. 7(a), (b), (c), (d), respectively. These figures show that the absolute cost and the relative cost saving decrease with increasing users' arrival time (departure time is fixed at 8 A.M.) while it increases with increasing users' departure time (arrival time is fixed at 5 P.M.). These results can be interpreted as follows. The relative cost saving would increase if EVs are available at home for a longer duration per day. This is because by connecting with the power grid longer, EVs can charge their batteries during off-peak hours and discharge energy to supply the HVAC system in on-peak hours more efficiently. However, EV parking time at home is directly related to users' arrival and departure times. Also, it is easy to recognize that by using V2G service, more cost saving would be achieved.

To investigate the impact of travel distance on the cost saving of the proposed scheme compared to the uncontrolled one, we fix the departure time ( 8 A.M.) and the arrival time (5 P.M.) and vary the travel distance of the EV. The EV battery SOC when it returns home depends on its energy consumption which, in turn, depends on the travel distance. Moreover, the electricity 


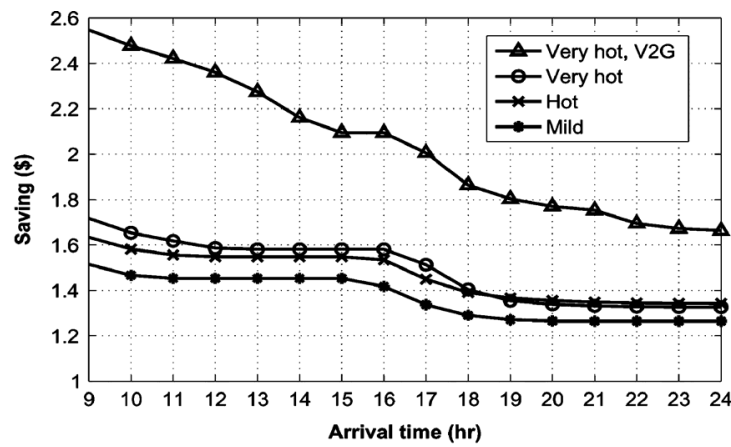

(a)

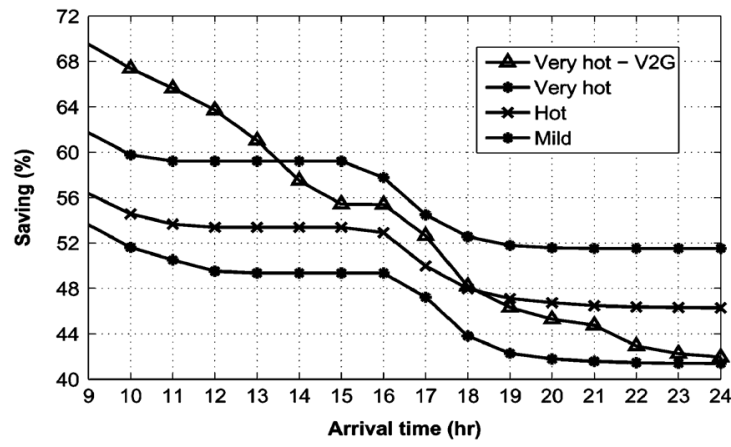

(b)

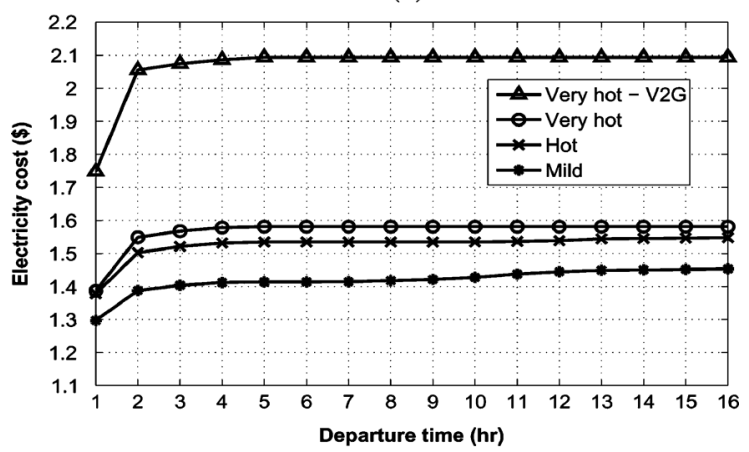

(c)

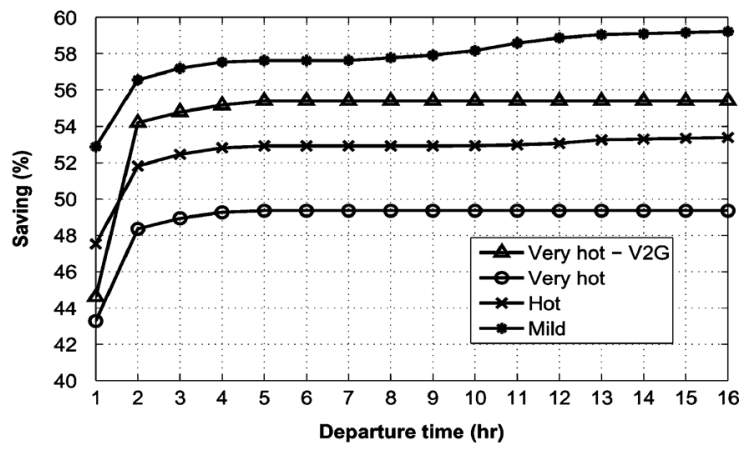

(d)

Fig. 7. Impact of departure time and arrival time on cost saving $\left(\delta=2{ }^{\circ} \mathrm{C}\right.$, $w=0.01 \$ /{ }^{\circ} \mathrm{C}$ ). (a) Varying arrival time (absolute). (b) Varying arrival time (relative). (c) Varying departure time (absolute). (d) Varying departure time (relative)

price is high around 5 P.M.; therefore, the higher SOC when EV gets home, the more energy can be discharged from the EV to supply power to the HVAC system, which consequently results in larger cost saving. The numerical results in Figs. 8(a), (b) confirm this point by showing that the cost saving decrease as the travel distance increases.

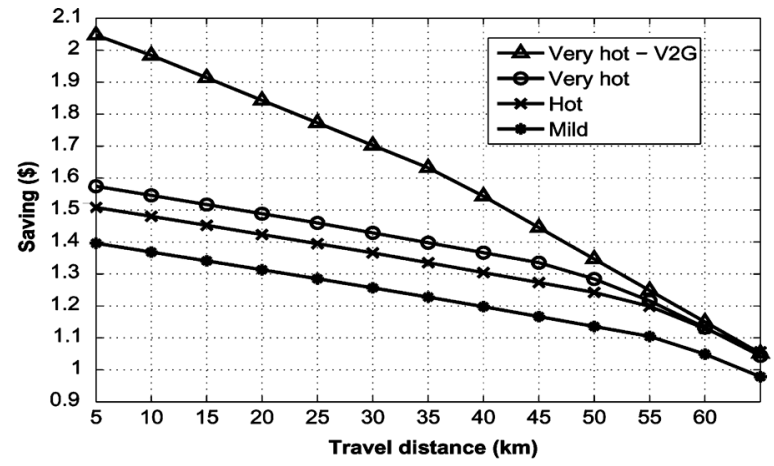

(a)

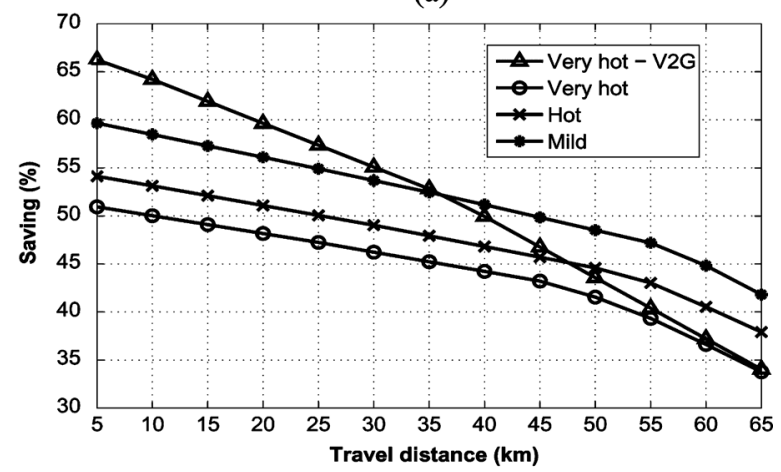

(b)

Fig. 8. Impact of travel distance on cost saving (very hot, $\delta=2{ }^{\circ} \mathrm{C}, w=$ $0.01 \$ /{ }^{\circ} \mathrm{C}$ ) (a) Absolute saving. (b) Relative saving.

\section{B. Multiple-House Scenario}

In the previous section, we have shown the strength of our proposed control scheme for the single-house scenario compared to the optimal scheme without discharging and the uncontrolled one. In this section, we will demonstrate that, it is even more cost-efficient if we apply our proposed control scheme to manage a group of households. Toward this end, we consider a community of 100 households $(M=100)$. We also assume that all EVs are Nissan Leaf whose specifications such as battery capacity, maximum charging/discharging power are described in the previous section. We take building thermal parameters from [23] as mean values for thermal parameters of houses in the community. Each thermal parameter (resistance and capacitance) of each house is chosen to be uniformly distributed in the interval of $\pm 20 \%$ around the mean value to represent the diversify of houses in the community. In practice, the size of $\mathrm{AC} /$ heater units would be chosen based on the size and shape of the building. To capture the variety of HVAC systems, we assume that the power rating and COP of HVAC units in the community are uniformly distributed in $[4 \mathrm{~kW}, 6 \mathrm{~kW}]$ and $[2.5,3.5]$ intervals. We assume that all households are occupied all day and the desired indoor temperature for all households is $23^{\circ} \mathrm{C}$ in the summer. The initial indoor temperature is set randomly in $[22,24]^{\circ} \mathrm{C}$ interval and the maximum imported power from the grid is set equal to $1 \mathrm{MW}$. The initial SOC of EVs are chosen to be uniformly distributed in the range $\left[S O C^{\min }, S O C^{\max }\right]$.

The travel patterns of EVs in the community are randomly generated based on statistical data from National Household Travel Survey (NHTS) data set, which collects daily travel information of households in U.S. [30]. For simplicity, only the departure time of the first trip and the arrival time of the last 


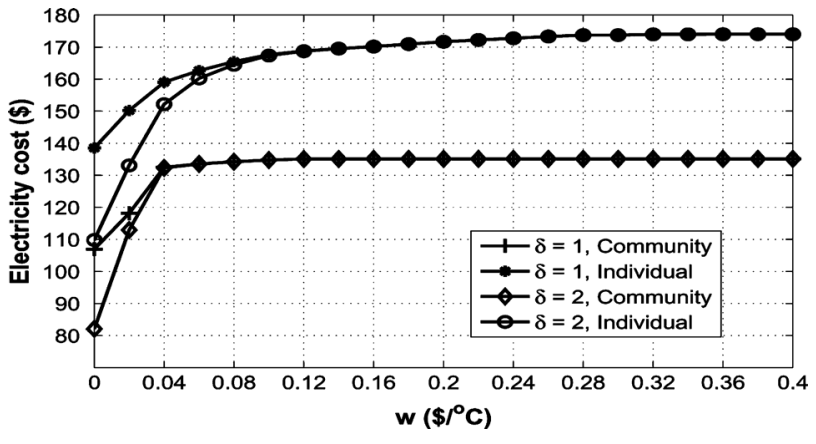

Fig. 9. Comparison optimal electricity cost under community-based optimal and individual-based optimal solutions (No V2G).

trip are taken into account even though our proposed model can cover multiple trips per day. This assumption can be justified because if an EV comes back home for a short time during the day, its available time for charging/discharging between the trips is small and the benefit due to the energy exchange would be insignificant. We choose the departure times for different EVs randomly according to a normal distribution with the mean of 7 A.M. and the standard deviation of 2 hours. The arrival time is drawn randomly according to another normal distribution with the mean of 6 P.M. and the standard deviation of 2 hours. These parameter settings were suggested by [42], which were established by using the data set given in [30]. Daily travel distance also follows a log-normal distribution with the mean of 32 miles and a standard deviation of 24 miles [41]. Based on EV ownership information [30], we assume that there are 9 households which have no EV, 32 households each of which has $1 \mathrm{EV}, 36$ households each of which has 2 EVs, 12 households with 3 EVs each, and 11 households each of which has 4 EVs. Therefore, there are $184 \mathrm{EVs}$ under consideration. There are about 35\% of vehicles do not travel all day according to [14], [17], [30]; therefore, we set 64 EVs out of the 184 EVs to be available at home all day. For remaining EVs, their travel patterns (departure time, arrival time, and travel distance) follow the normal and lognormal distributions as described above.

To ease the analysis and presentation, we assume that all households choose the same value of $w_{k}=w$ and same the value of $\delta_{k}=\delta$ in our model. We evaluate the performance for two cases where the proposed optimization framework is applied for the whole community with 100 households and for individual households, respectively. The results corresponding to these two cases are indicated as "Community" and "Individual" in Figs. 9, 10(a), respectively. Fig. 9 shows the optimal costs for both control schemes in the very hot summer day. It can be observed that the total electricity cost is reduced quite significantly when we optimize the energy usage for the whole community compared to the case when each household optimizes its energy consumption separately. The performance gain is about $20 \%$.

Fig. 10(a) plots the power imported from the grid for a hot summer day and $w=0.02 \$ /{ }^{\circ} \mathrm{C}$. Fig. $10(\mathrm{~b})$ illustrates the total power consumption and charging/discharging due to ACs and EVs in the community. As can be seen, the total energy imported from the grid in the community-optimization scheme is zero during peak hours (1 P.M. to 6 P.M.). When each household applies our optimal scheme separately, the total energy imported from the grid is non-zero for several high-price hours.

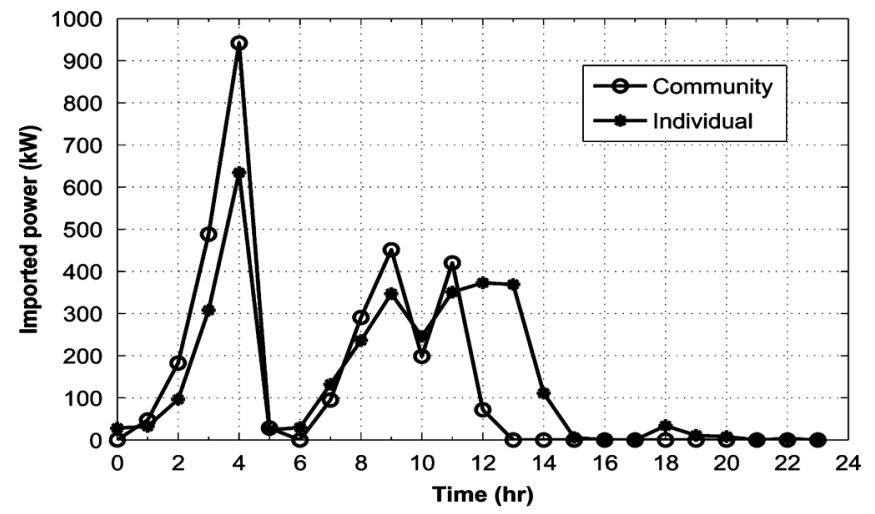

(a)

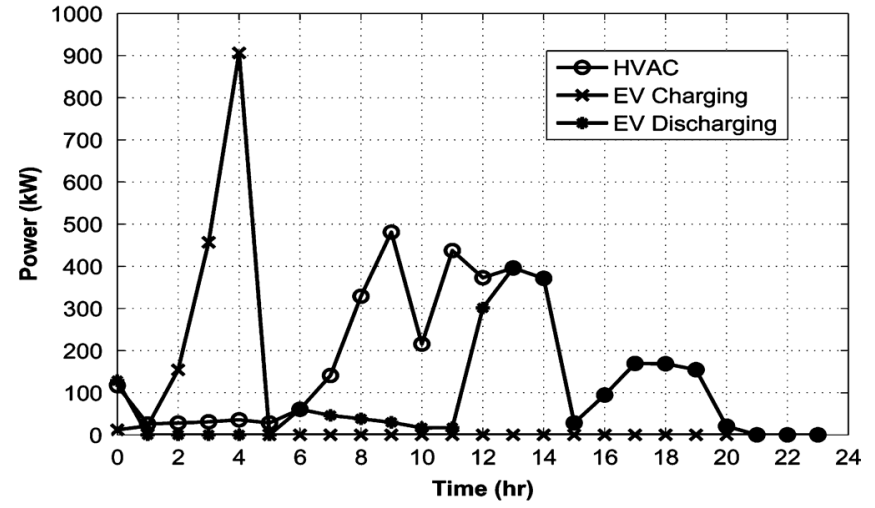

(b)

Fig. 10. Imported power and power consumption, charging/discharging (very hot day, no V2G, $w=0.02 \$ /{ }^{\circ} \mathrm{C}, \delta=2{ }^{\circ} \mathrm{C}$ ). (a) Imported power. (b) Power consumption, charging/discharging (community).

This "demand response" effect achieved by the proposed community-optimization scheme is very desirable since it helps reduce the peak demand in on-peak hours. In addition, Fig. 10(b) shows that by exploiting EV discharging capability we can reduce the demand and save the electricity cost during high-price hours.

\section{CONCLUSION}

We have proposed a unified framework to jointly optimize the EV and home energy scheduling considering user comfort preference. The proposed control model captures different key modeling aspects including thermal dynamics, EV travel, and user occupancy patterns, as well as operational constraints of the HVAC system and EVs. We have presented extensive numerical results to demonstrate the impacts of different parameters on the electricity cost, the significant gain achieved by the proposed model, and benefits of optimization of EV and home energy scheduling for multiple houses in a residential community.

\section{REFERENCES}

[1] U.S. Environmental Protection Agency [Online]. Available: www.epa. gov/greenbuilding/pubs/gbstats.pdf

[2] S. Shao, M. Pipattanasomporn, and S. Rahman, "Demand response as a load shaping tool in an intelligent grid with electric vehicles," IEEE Trans. Smart Grid, vol. 2, no. 4, pp. 624-631, Dec. 2011.

[3] A.-H. Mohsenian-Rad and A. Leon-Garcia, "Optimal residential load control with price prediction in real-time electricity pricing environments," IEEE Trans. Smart Grid, vol. 1, no. 2, pp. 120-133, Sep. 2010. 
[4] P. Constantopoulos, F. Schweppe, and R. Larson, "ESTIA: A real-time consumer control scheme for space conditioning usage under spot electricity pricing," Comput. Oper. Res., vol. 19, no. 8, pp. 751-765, 1991.

[5] M. C. Mozer, L. Vidmar, and R. H. Dodier, , M. C. Mozer, M. I. Jordan, and T. Petsche, Eds., "The neurothermostat: Adaptive control of residential heating systems," in Advances in Neural Information Processing Systems 9. Cambridge, MA, USA: MIT Press, 1997.

[6] Y. Liang, D. I. Levine, and Z.-J. Shen, "Thermostats for the smart grid: Models, benchmarks, and insights," Energy J., vol. 33, no. 4, Mar. 2012.

[7] P. Du and N. Lu, "Appliance commitment for household load scheduling," IEEE Trans. Smart Grid, vol. 2, no. 2, pp. 411-419, June 2011.

[8] J. Kondoh, N. Lu, and D. J. Hammerstrom, "An evaluation of the water heater load potential for providing regulation service," IEEE Trans. Power Syst., vol. 26, no. 3, pp. 1309-1316, Aug. 2011.

[9] A. Y. Saber and G. K. Venayagamoorthy, "One million plug-in electric vehicles on the road by 2015," in Proc. IEEE Conf. Intell. Transp. Syst., Oct. 2009.

[10] R. C. Green, II, L. Wang, and M. Alam, "The impact of plug-in hybrid electric vehicles on distribution networks: A review and outlook," Renewable Sustain. Energy Rev., vol. 15, no. 1, pp. 544-553, Jan. 2011.

[11] S. W. Hadley and A. A. Tsvetkova, "Potential impacts of plug-in hybrid electric vehicles on regional power generation," Electr. J., vol. 22, no. 10, pp. 56-68, Dec. 2009.

[12] K. Clement-Nyns, E. Haesen, and J. Driesen, "The impact of charging plug-in hybrid electric vehicles on a residential distribution grid," IEEE Trans. Power Syst., vol. 25, no. 1, pp. 371-380, Feb. 2010.

[13] T.-K. Lee, Z. Bareket, T. Gordon, and Z. S. Filipi, "Stochastic modeling for studies of real-world PHEV usage: Driving schedule and daily temporal distributions," IEEE Trans. Veh. Tech., vol. 61, no. 4, pp. 1493-1502, May 2012.

[14] D. Wu, D. C. Aliprantis, and K. Gkritza, "Electric energy and power consumption by light-duty plug-in electric vehicles," IEEE Trans. Power Syst., vol. 26, no. 2, pp. 738-746, May 2011.

[15] A. Ashtari, E. Bibeau, S. Shahidinejad, and T. Molinski, "PEV charging profile prediction and analysis based on vehicle usage data," IEEE Trans. Smart Grid, vol. 3, no. 1, pp. 341-350, Mar. 2012.

[16] N. Rotering and M. Ilic, "Optimal charge control of plug-in hybrid electric vehicles in deregulated electricity markets," IEEE Trans. Power Syst., vol. 26, no. 3, pp. 1021-1029, Aug. 2011.

[17] D. Wu, D. C. Aliprantis, and L. Ying, "Load scheduling and dispatch for aggregators of plug-in electric vehicles," IEEE Trans. Smart Grid, vol. 3, no. 1, pp. 368-376, Mar. 2012.

[18] O. Sundstrom and C. Binding, "Flexible charging optimization for electric vehicles considering distribution grid constraints," IEEE Trans. Smart Grid, vol. 3, no. 1, pp. 26-37, Mar. 2012.

[19] S. Beer, T. Gomez, D. Dallinger, T. Momber, C. Marnay, M. Stadler, and J. Lai, "An economic analysis of used electric vehicle batteries integrated into commercial building microgrids," IEEE Trans. Smart Grid, vol. 3, no. 1, pp. 517-525, Mar. 2012.

[20] J. Lassila, J. Haakana, V. Tikka, and J. Partanen, "Methodology to analyze the economic effects of electric cars as energy storages," IEEE Trans. Smart Grid, vol. 3, no. 1, pp. 506-516, Mar. 2012.

[21] A. Molina-Garciá, M. Kessler, J. A. Fuentes, and E. Gómez-Lázaro, "Probabilistic characterization of thermostatically controlled loads to model the impact of demand response programs," IEEE Trans. Power Syst., vol. 26, no. 1, pp. 241-251, Feb. 2011.

[22] Yi Zong, D. Kullmann, A. Thavlov, O. Gehrke, and H. W. Bindner, "Application of model predictive control for active load management in a distributed power system with high wind penetration," IEEE Trans. Smart Grid, vol. 3, no. 2, pp. 1055-1062, Jun. 2012.

[23] A. Thavlov, "Dynamic optimization of power consumption," M.S. thesis, Tech. Univ. Denmark, Kongens Lyngby, Denmark, 2008.

[24] H. Madsen and J. Holst, "Estimation of continuous-time models for the heat dynamics of a building," Energy Buildings, vol. 22, no. 1, pp. 67-79, Mar. 1995

[25] P. Bacher and H. Madsen, "Identifying suitable models for the heat dynamics of buildings," Energy Buildings, vol. 43, no. 7, pp. 1511-1522, Jul. 2011.

[26] D. Bargiotas and J. D. Birdwell, "Residential air conditioner dynamic model for direct load control," IEEE Trans. Power Del., vol. 3, no. 4, pp. 2119-2126, Oct. 1988.
[27] J. Siroký, F. Oldewurtel, J. Cigler, and S. Prívara, "Experimental analysis of model predictive control for an energy efficient building heating system," Appl. Energy, vol. 88, no. 9, pp. 3079-3087, Sep. 2011.

[28] R. Halvgaard, N. K. Poulsen, H. Madsen, and J. B. Jorgensen, "Economic model predictive control for building climate control in a smart grid," in Proc. IEEE PES Innov. Smart Grid Technol. (ISGT), Jan. 2012, pp. 1-6.

[29] T. S. Pedersen, P. Andersen, K. M. Nielsen, H. L. Starmose, and P. D. Pedersen, "Using heat pump energy storage in the power grid," in Proc. IEEE Int. Conf. Control Appl. (CCA), Sep. 2011, pp. 1106-1111.

[30] U.S. Department of Transportation, 2009, National Household Travel Survey [Online]. Available: http://nhts.ornl.gov/index.shtml

[31] [Online]. Available: http://www.nissanusa.com/leaf-electric-car/index

[32] S. Boyd and L. Vandenberghe, Convex Optimization. Cambridge, U.K.: Cambridge Univ. Press, 2004.

[33] E. F. Camacho and C. B. Alba, Model Predictive Control, 2nd ed. London, U.K.: Springer-Verlag, 2004.

[34] Weather website [Online]. Available: http://www.wunderground.com/ history

[35] [Online]. Available: http://rredc.nrel.gov/solar/old_data/nsrdb/19912010/hourly/siteonthefly.cgi?id $=722265$

[36] [Online]. Available: http://pjm.com/markets-and-operations.aspx

[37] CVX solver [Online]. Available: http://cvxr.com/cvx

[38] [Online]. Available: http://www.nissan-global.com/EN/TECHNOLOGY/OVERVIEW/leaf to home.html

[39] [Online]. Available: http://www.eia.gov/consumption/residential/reports/2009/square-footage.cfm

[40] [Online]. Available: http://www.energystar.gov/?c=roomac.pr_properly_sized

[41] W. Su and M. Y. Chow, "Performance evaluation of an EDA-based large-scale plug-in hybrid electric vehicle charging algorithm," IEEE Trans. Smart Grid, vol. 3, no. 1, pp. 308-315, Mar. 2012.

[42] C. Jin, J. Tang, and P. Ghosh, "Optimizing electric vehicle charging with energy storage in the electricity market," IEEE Trans. Smart Grid, vol. 4, no. 1, pp. 311-320, Mar. 2013.

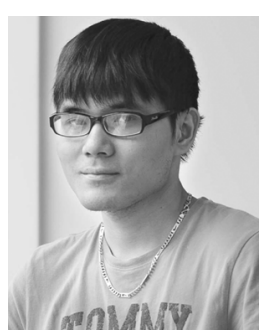

Duong Tung Nguyen received the B.Eng. from Hanoi University of Technology, Vietnam, in 2011. $\mathrm{He}$ is currently a Graduate Student at the Institut National de la Recherche Scientifique (INRS), Université du Québec, Montréal, QC, Canada. His research lies in the smart grids area with current focus on home energy management, charging optimization for electric vehicles, and control and optimization issues for microgrids.

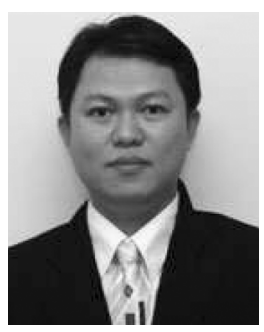

Long Bao Le (S'04-M'07-SM'12) received the B.Eng. (with Highest Distinction) degree from Ho Chi Minh City University of Technology, Vietnam, in 1999, the M.Eng. degree from Asian Institute of Technology, Pathumthani, Thailand, in 2002, and the Ph.D. degree from the University of Manitoba, Winnipeg, MB, Canada, in 2007.

From 2008 to 2010 , he was a Postdoctoral Research Associate with Massachusetts Institute of Technology, Cambridge, MA, USA. Since 2010, he has been an assistant professor with the Institut National de la Recherche Scientifique (INRS), Université du Québec, Montréal, QC, Canada, where he leads a research group working on smartgrids, cognitive radio and dynamic spectrum sharing, radio resource management, network control and optimization for wireless networks.

Dr. Le is a member of the editorial board of IEEE Communications Surveys and Tutorials and IEEE Wireless Communications Letters. He has served as technical program committee co-chair of the Wireless Networks track at IEEE VTC2011-Fall and the Cognitive Radio and Spectrum Management track at IEEE PIMRC2011. 